\title{
Structural, Morphological and Optical Properties of Gold Nanoparticles Using Laser Ablation in Liquid for Sensor Applications
}

\author{
Zainab H. Tawfiq ${ }^{1,2}$, Sallah A. Adnan ${ }^{1, a}$, Makram A. Fakhri ${ }^{1, b}$, Rihab K. Hamad ${ }^{1}$ \\ ${ }^{1,2,3}$ Laser and Optoelectronic department, University of technology, Baghdad, Iraq. \\ ${ }^{4}$ Al esraa university college, Computer engineering techniques department, Baghdad, Iraq. \\ Salahadnan9999@yahoo.com, mokaram_76@yahoo.com,140017@uotechnology.edu.iq
}

\begin{abstract}
The impact of laser wavelength on gold nanoparticle (Au NPs) creation is accounted by the extraction of gold atoms from the gold specimen immersed in ethanol. Pulsed Laser ablation in fluid procedure of the gold target was carried out by employing Nd:YAG laser (nanosecond pulses). Characterization of accomplished gold NPs was achieved by Atomic Force Microscopy AFM), X-Ray Diffraction (XRD) and UltravioletVisible (UV-VIS) absorption and transmission spectroscopy. The outcomes demonstrated the attributes of the prepared NPs, contingent upon XRD, AFM. NPs gotten by $532 \mathrm{~nm}$ laser have preferred properties over that accomplished by $1064 \mathrm{~nm}$ laser as indicated by the highest values of intensity of shorter wavelengths.
\end{abstract}

Index Terms - Laser ablation in liquid; optical preparation; gold nanoparticles; Refractive Index Sensors.

\section{INTRODUCTION}

Nanoparticles (Nps) of respectable metals have, as of late turned out, to be intriguing materials in light of their properties and imperative applications in numerous scientific fields [1]. The manufacture of metallic nanoparticles (NPs), for the most part gold and silver, has pulled in much enthusiasm because of their surface plasmon resonance related properties that are conceivably valuable for their biological applications [2].

The most significant issues in AuNP creation are the control of size, shape, and surface functionalization [3]. Because of their chemical stability and conspicuous optical properties, gold nanoparticles (Au-NPs) have been at the focal point of consideration in nanomedicine [4].

Au-NPs can likewise give the difference to malignant growth imaging through photoacoustic or light dispersing, just as structure versatile surface-upgraded raman dissipating based labels for disease focusing on and conclusion.Nanotechnology stresses materials in 10-9 meter scales, including biotechnology, material sciences, PC sciences, prescriptions, drug store and designing. Nanotechnology initially appeared in ninth century by Mesopotamian individuals for giving glossy impact in pots.

In 1857, Michael Faraday for the first time found the ruby gold nanoparticles (AuNPs), which turned into the establishment for the advanced nanotechnology [5]. Different structural and morphological characters of the created NPs can be constrained by laser fluence, spot estimate, wavelength, pulse width, and repetition rate of laser pulse [6].

The laser removal of a strong target inundated in a fluid milieu has turned into an inexorably imperative "top down" strategy for manufacturing nanostructured materials[7].In this strategy, NP creation results from removal of a strong target put in a fluid medium. Changing the laser parameters and the idea of the encompassing fluid medium empowers control of size, focus, chemical composition and utilitarian properties of the NPs.Numerous laser types have been 
proposed for this reason, including nano, pico and femtosecond lasers at different wavelengths running from infra-red $(1064 \mathrm{~nm})$ to ultraviolet $(248 \mathrm{~nm})$ [8].Another technique dependent on laser removal in fluid of the mass metal showed up over the most recent couple of years for the amalgamation of gold nanoparticles [3]. Mafune' et al. have demonstrated that it is likewise conceivable to get great control of the elements of the nanoparticles by utilizing the second harmonic of a Nd:YAG laser $(532 \mathrm{~nm})$ and a surfactant like sodium dodecysulfate (SDS).Uhlirand Turner was the first who labelled the construction of porous silicon coating (PSLs) on silicon electrodes in hydrofluoric acid electrolytes under anodic bias.

The diameter of PSL pores or channels was found to extend from 1 up to $100 \mathrm{~nm}$ related with porosities of $20-80 \%$ [9]. Since permeable silicon can be effectively manufactured legitimately from a similar single-crystal silicon wafers utilized in the microelectronics business, it appeared to be perfect for a Si-based optoelectronic innovation (the mix of optics and light emanation with standard silicon-based microelectronic gadgets). The benefit of a silicon-based EL gadget is that it would preferably be well matched with well-known strong state advancements that utilization silicon, and stay away from the cost and perils related with the treatment of $\mathrm{III} \pm \mathrm{V}$ semiconductors. Porous silicon is an all-around described, flexible inorganic material created through a galvanostatic, synthetic, or photochemical drawing methodology within the sight of HF. Contingent on the carving conditions, Porous silicon by and large has an intricate, anisotropic nanocrystalline architecture of high surface area $[10,11]$.

\section{EXPERIMENTAL WORK}

In order to extract gold nanoparticles (NP), the first step, inundated $2 \mathrm{~mm}$ diameter of circular Hugh purity gold alloy in $5 \mathrm{ml}$ of ethanol. after that step irradiate the metal using pulsed Q-Switched NdYAG laser of $532 \mathrm{~nm}$, by applying an energy of $1000 \mathrm{~mJ} /$ pulse and 100 pulses with a $12 \mathrm{~cm}$ lens (spot size: $1 \mathrm{~mm}^{2}$ ) onto sample in order to remove the NPs as illustrated in Figure 1. commonly the red coloration of the solution leads to the creation of gold NPs after few seconds of removal.

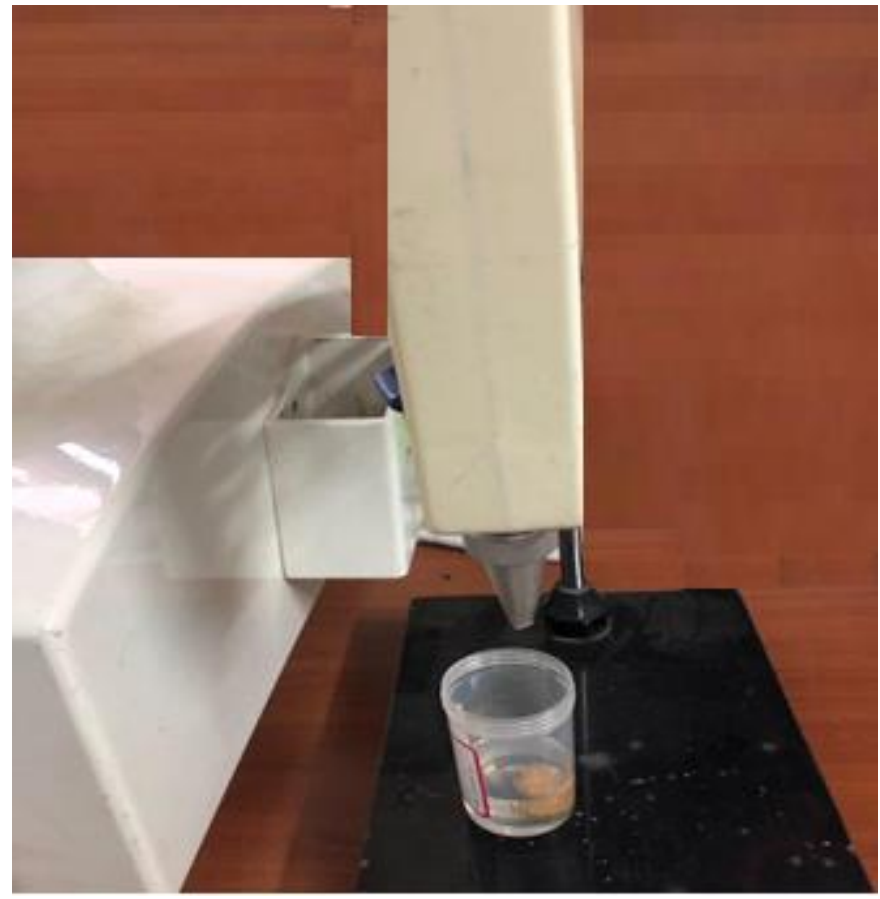

FIG. 1. EXPERIMENTAL SET UP FOR GOLD COLLOID PREPARATION BY LASER ABLATION IN SOLUTION. 
After the synthesis process, the prepared Ag NPs were considered utilizing UV-vis double beam spectrometer. The phase organization of the nanoparticles has been dictated by X-ray diffraction analysis utilizing a diffractometer system (x' pert pro MRD PW3040). Then again, Porous silicon was set up from silicon n-type and concentration $40 \%$ of hydrofluoric acid (HF) for 5 minutes engraving time and at $40 \mathrm{~mA}$ current. Finally, the gold blend has dropped on the porous silicon by drop casting technique which has subjected to heat so as to be dry.

\section{RESULT AND DISCUSSION}

Figure (2) demonstrates the XRD for the prepared gold NPs by PLA technique with 1064 and 532 $\mathrm{nm}$, and 100 pulses. Drop-casting technique was utilized for the purpose of gold NPs deposition on the porous silicon. Figure (2) shows the XRD of $532 \mathrm{~nm}$, three particular peaks were shown up at $2 \theta=34.05$, 38.15, 44.3. Basically,each one of the three peaks corresponded to the standard Bragg reflections (110), (111) and (200) of (fcc) lattice. Peak of 34.15 alludes to the $\mathrm{SiO} 2$ which showed up because of silicon collaboration with ethanol. At $1064 \mathrm{~nm}$, as appeared in Figure (2), two peaks showed up at $2 \theta=38.15$ and $2 \theta=44.3$, compared to the standard Bragg reflection (111) and (200). Because of the laser power impact, this made two phases to be shown up for gold, one at $532 \mathrm{~nm}$ and the other at $1064 \mathrm{~nm}$.

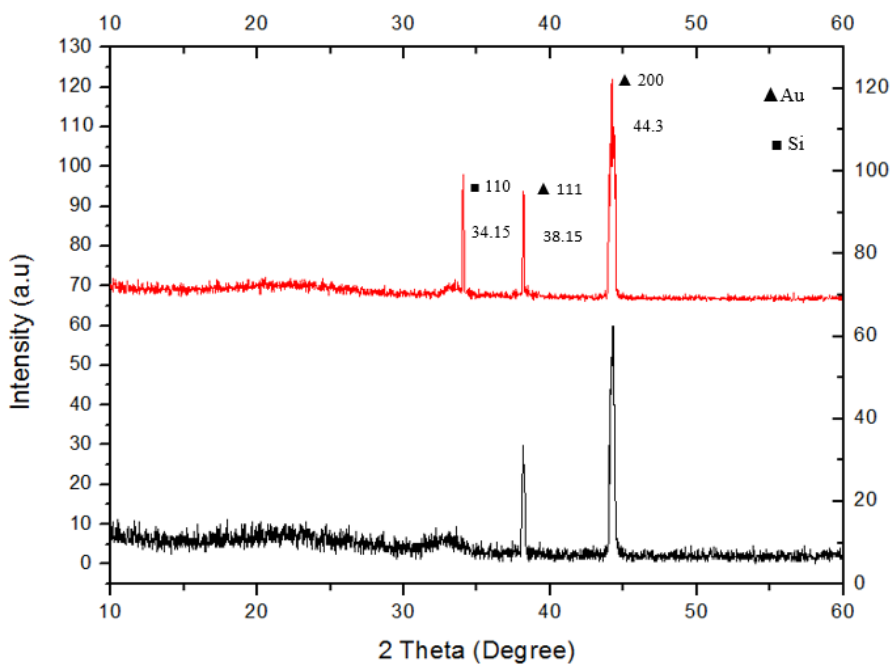

FIG.2. XRD ANALYSIS OF GOLD NANOPARTICLES

Figure (3.a.b) the morphological results are considered as a very interesting parameter for the photonics integrated circuits, optoelectronics and in the sensing applications. Both values of the root mean square and the grain size can be affected by the different wavelength of a pulsed laser. Fig. 3 (a and b) presented the topographic structure for gold NPs prepared at 532, $1064 \mathrm{~nm}$. At a $532 \mathrm{~nm}$ a small size of NPs was acquired, the surface roughness is (mean=18.034 nm, SD=12.807nm) and accomplished the good distribution and high smooth than that produced by $1064 \mathrm{~nm}$ which is (mean= $50.974 \mathrm{~nm}, \mathrm{SD}=40.792 \mathrm{~nm}$ ). This means that the gold interaction with $532 \mathrm{~nm}$ more than its ability of interaction with the other laser wavelength.

Figure (4.a.b) illustrated the SPR transmission and the absorption spectrum of the gold NPs as a function of wavelength for 1064 and $532 \mathrm{~nm}$ wavelengths. From this Figure one can observe that as the Au NPs size increase, the SPR band shifts to longer wavelengths. From Figure (4.a.), at 1064 nm, the gold NPs possess larger size than that obtained by $532 \mathrm{~nm}$ laser beam. As a result, the highest transmission peak has obtained by 1064, the transmission peaks obtained by 1064 and $532 \mathrm{~nm}$ where the transmission at $1064 \mathrm{~nm}$ about (89.07) and the effective transmission about $520 \mathrm{~nm}$ and the transmission at $532 \mathrm{~nm}$ are about (84.55) and the effective transmission about $513 \mathrm{~nm}$, this value gives 
an indication that when minimizing the Laser wavelengths to ablated the gold nanoparticles, the result will be a blue shift and this means that the grain size will be less. From Figure (4.b.), the highest peak of absorption resulted by the application of $1064 \mathrm{~nm}$ laser beam and the effective absorption is about 0.49 , while when using $532 \mathrm{~nm}$ laser, the absorption peak obtained at $530 \mathrm{~nm}$ and the effective absorption is about 0.70 . The absorption of SPR of gold NPs depends on the size and shape of gold NPs when enlarging the volume, then minimizing the absorption in order to give maximum absorption from $500 \mathrm{~nm}$ to the near infrared area of the spectrum, surface plasmon resonance can be tuned easily. For example, Cytodiagnostics spherical colloidal gold in the area between 515-570nm has the maximum absorbance as demonstrated above, however the irregular form particles like gold nanorods, and urchin form gold nanoparticles (also known as gold nanostars) have the maximum absorbance in the nearinfrared area of the spectra This is similar to the results recorded by Nguyen Ngoc Long et al [12].

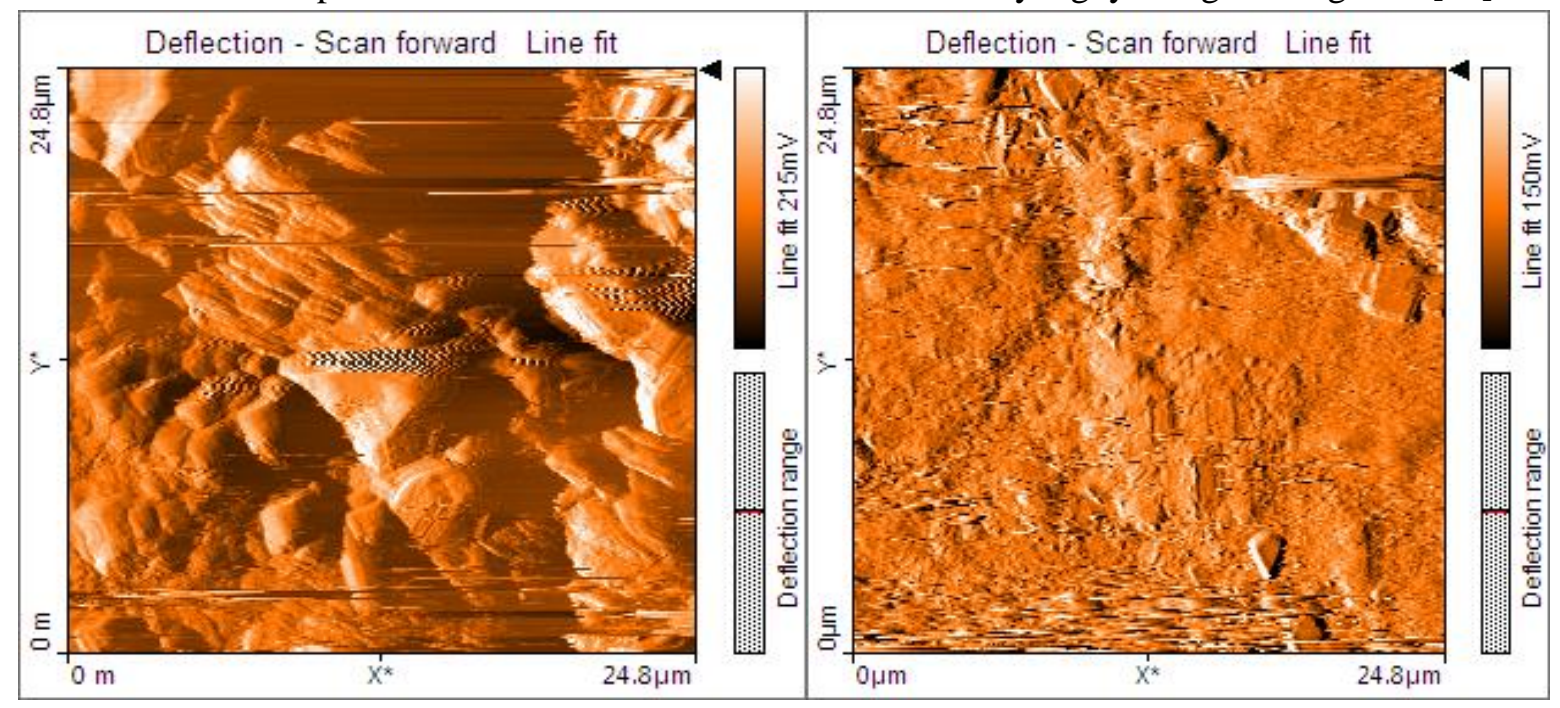

Fig.3.A. AfM ANALYSIS OF GOLD NANOPARTICLES AT 532 NM, (B) AFM ANALYSIS OF GOLD NANOPARTICLES AT 1064 NM

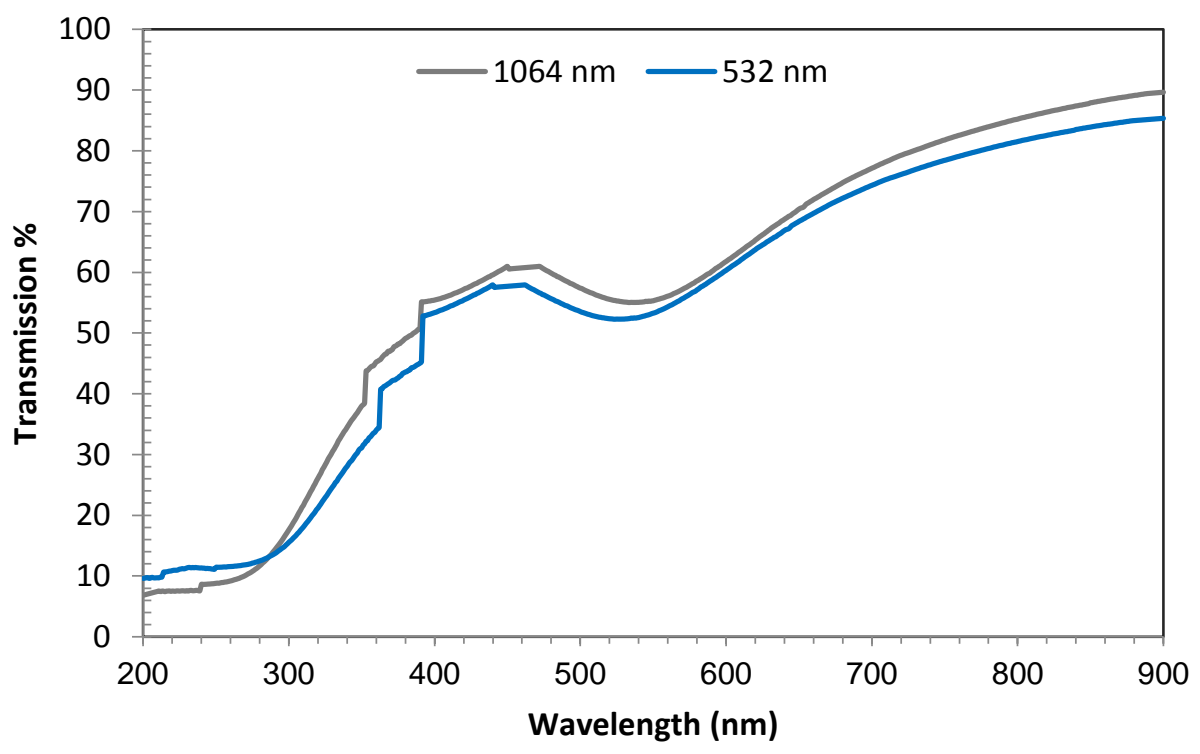

FIG.4.A..UV-VIS TRANSMISSION SPECTRUM FOR 1064 AND 532 NM LASERS ON THE GOLD NPS EMBEDDED IN ETHANOL. 


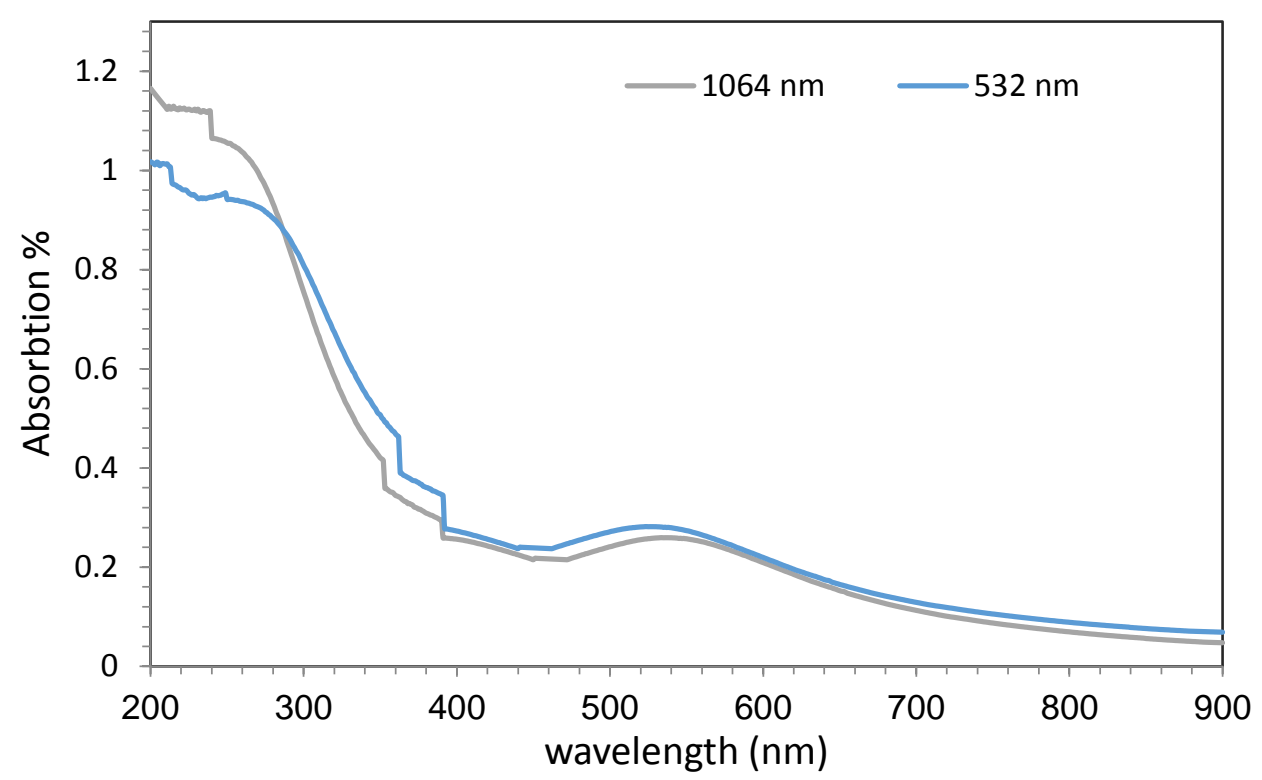

FIG.4.B. UV-VIS ABSORPTION SPECTRUM FOR 1064 AND 532 NM LASERS ON THE GOLD NPS EMBEDDED IN ETHANOL.

\section{CONCLUSION}

Gold NPs mixed in ethanol were prepared by PLA technique utilizing a nanosecond Q-switched Nd:YAG pulsed laser at $1064 \mathrm{~nm}$ and $532 \mathrm{~nm}$ laser. Drop casting technique was utilized to sedimentation the gold NPs on the permeable silicon. The results have demonstrated characteristics of prepared NPs, contingent upon XRD, AFM.NPs acquired by $532 \mathrm{~nm}$ laser own better properties over that accomplished by $1064 \mathrm{~nm}$ laser as indicated by the most intensity of shorter wavelengths. As result, the gold NPs interaction increases, as the number of laser pulses increases. From AFM results, two phases of gold were shown up at $532 \mathrm{~nm}$, while only one phase was shown up at $1064 \mathrm{~nm}$. One can conclude that as the Au NPs size increased, the SPR band shifts to longer wavelengths.

\section{REFERENCES}

[1] HOJABRI, Alireza, Fatemeh Hajakbari, and Maryam Debashi Shoreh. "Characterization of silver nanoparticles prepared by laser ablation in distilled water." NANO CON, Nov 5th-7th, Brno, Czech Republic, EU (2014).

[2] D. Riabinina, J. Zhang, M. Chaker, and D. Ma, "Size Control of Gold Nanoparticles Synthesized by Laser Ablation in Liquid Media," ISRN Nanotechnology, $2012 \quad$ (2012) Article ID 297863, 5 pages http://dx.doi.org/10.5402/2012/297863

[3] V. Amendola, S. Polizzi, and M. Meneghetti, "Laser Ablation Synthesis of Gold Nanoparticles in Organic Solvents," Phys. Chem. B 110(14) 7232-7237, 2006

[4] F. Correard, K. Maximova, M. Estève, C. Villard, M. Roy, A. Al-Kattan, M. Sentis, M. Gingras, Andrei V Kabashin, and D. Braguer, "Gold nanoparticles prepared by laser ablation in aqueous biocompatible solutions: assessment of safety and biological identity for nanomedicine applications," Int J Nanomedicine. 2014; 9: 54155430 .

[5] M. Das, Kyu H. Shim, Seong S. A. An, Dong K.Yi,, "Review on Gold Nanoparticles and Their Applications," Toxicology and Environmental Health Sciences, 3(4), 193-205, 2011

[6] N. Mirghassemzadeh, M. Ghamkhari, and D. Dorranian, "Dependence of Laser Ablation Produced Gold Nanoparticles Characteristics on the Fluence of Laser Pulse," Soft Nanoscience Letters, 2013(3) 101-106, 2013.

[7] J. Sylvestre, S. Poulin, Andrei V. Kabashin, E. Sacher, M. Meunier, John H. T. Luong, "Surface Chemistry of Gold Nanoparticles Produced by Laser Ablation in Aqueous Media," J. Phys. Chem. B, 108(43) 16864-16869. 2004.

[8] D. Riabinina and M. Chaker, "Dependence of gold nanoparticle production on pulse duration by laser ablation in liquid media," Nanotechnology 23(13) 135603, 2012.

[9] V. Lehmann and U. Gösele, "Porous silicon formation : A quantum wire effect Porous silicon formation : A quantum," Appl. Phys. Lett. 58, $856,1991$. 
[10] B. M. P. Stewart and J. M. Buriak, "Chemical and Biological Applications of Porous Silicon Technology " Adv. Mater, 12(12) 859-869, 2000.

[11] Zainab H Tawfiq, Makram A Fakhri, Salah A Adnan," Photonic Crystal Fibres PCF for Different Sensors in Review," IOP Conference Series: Materials Science and Engineering, 454(1) 012173, 2018.

[12] C. Wang and T. Wang, "Synthesis and optical properties of colloidal gold nanoparticles Synthesis and optical properties of colloidal gold nanoparticles,” J. Phys. Conf. Ser, 187(1). 012026, 2009. 